\title{
Duration of antibiotic therapy in critically ill patients: a randomized controlled trial of a clinical and C-reactive protein-based protocol versus an evidence-based best practice strategy without biomarkers
}

Isabela Borges ${ }^{1,2^{*}}$ (D), Rafael Carneiro ${ }^{1}$, Rafael Bergo ${ }^{1}$, Larissa Martins ${ }^{3}$, Enrico Colosimo $^{3}$, Carolina Oliveira ${ }^{4}$, Saulo Saturnino ${ }^{4}$, Marcus Vinícius Andrade ${ }^{4}$, Cecilia Ravetti ${ }^{1}$, Vandack Nobre ${ }^{1}$, on behalf of NIIMI - Núcleo Interdisciplinar de Investigação em Medicina Intensiva

\begin{abstract}
Background: The rational use of antibiotics is one of the main strategies to limit the development of bacterial resistance. We therefore sought to evaluate the effectiveness of a C-reactive protein-based protocol in reducing antibiotic treatment time in critically ill patients.
\end{abstract}

Methods: A randomized, open-label, controlled clinical trial conducted in two intensive care units of a university hospital in Brazil. Critically ill infected adult patients were randomly allocated to (i) intervention to receive antibiotics guided by daily monitoring of CRP levels and (ii) control to receive antibiotics according to the best practices for rational use of antibiotics.

Results: One hundred thirty patients were included in the CRP $(n=64)$ and control $(n=66)$ groups. In the intention-to-treat analysis, the median duration of antibiotic therapy for the index infectious episode was 7.0 (5.08.8) days in the CRP and $7.0(7.0-11.3)$ days in the control $(p=0.011)$ groups. A significant difference in the treatment time between the two groups was identified in the curve of cumulative suspension of antibiotics, with less exposure in the CRP group only for the index infection episode $(p=0.007)$. In the per protocol analysis, involving 59 patients in each group, the median duration of antibiotic treatment was 6.0 (5.0-8.0) days for the CRP and $7.0(7.0-10.0)$ days for the control $(p=0.011)$ groups. There was no between-group difference regarding the total days of antibiotic exposure and antibiotic-free days.

(Continued on next page)

\footnotetext{
* Correspondence: isabelanborges@gmail.com

${ }^{1}$ Graduate Program in Health Sciences: Infectious Diseases and Tropical

Medicine, Department of Internal Medicine, School of Medicine and Hospital

das Clínicas, Universidade Federal de Minas Gerais, Belo Horizonte, Brazil

${ }^{2}$ Departamento de Clínica Médica, $2^{\circ}$ andar Faculdade de Medicina. Av. Alfredo Balena, 190, Santa Efigênia, Belo Horizonte, Minas Gerais, Brazil

Full list of author information is available at the end of the article
}

C C The Author(s). 2020 Open Access This article is licensed under a Creative Commons Attribution 4.0 International License, which permits use, sharing, adaptation, distribution and reproduction in any medium or format, as long as you give appropriate credit to the original author(s) and the source, provide a link to the Creative Commons licence, and indicate if changes were made. The images or other third party material in this article are included in the article's Creative Commons licence, unless indicated otherwise in a credit line to the material. If material is not included in the article's Creative Commons licence and your intended use is not permitted by statutory regulation or exceeds the permitted use, you will need to obtain permission directly from the copyright holder. To view a copy of this licence, visit http://creativecommons.org/licenses/by/4.0/ The Creative Commons Public Domain Dedication waiver (http://creativecommons.org/publicdomain/zero/1.0/) applies to the data made available in this article, unless otherwise stated in a credit line to the data. 
(Continued from previous page)

Conclusions: Daily monitoring of CRP levels may allow early interruption of antibiotic therapy in a higher proportion of patients, without an effect on total antibiotic consumption. The clinical and microbiological relevance of this finding remains to be demonstrated.

Trial registry: ClinicalTrials.gov Identifier: NCT02987790. Registered 09 December 2016.

Keywords: Sepsis, Infection, Critical illness, Antibiotic, Antibiotic stewardship, C-reactive protein

\section{Background}

Increasing concerns about antimicrobial abuse and development of bacterial resistance have fueled the search for the objective and rational use of antibiotics. There is growing evidence supporting the use of shorter antibiotic courses to treat various types of infection, with clinical outcomes similar to those obtained with longer treatments [1-6]. Individualization of antibiotic treatment time has been gaining importance [7-10]. This measure prevents unnecessary exposure to antibiotics while reducing the risk of therapeutic failure in those with a late response.

Circulating inflammatory biomarkers have been used as a guide to support treatment individualization. One useful marker is procalcitonin (PCT), whose benefit in reducing antibiotic treatment time was demonstrated in several studies [11-16], including a potential reduction in the mortality of critically ill patients [14-16]. Nevertheless, the high cost of PCT testing reduces its availability in some settings $[10,17]$. In this context, C-reactive protein (CRP) may be a reasonable low-cost alternative $[10,16,18]$. A recent meta-analysis demonstrated that the PCT-guided algorithms only showed a survival benefit when used in combination with CRP, along with other specificities [16]. Nevertheless, few studies testing a CRP-guided strategy have been conducted in adult critical ill patients. A recent single-center clinical trial involving patients with sepsis suggested that CRP may be a useful marker to guide antibiotic treatment time, when compared to a PCT strategy [19].

The objective of this study was to test the impact of a decision flowchart based on CRP serum levels and clinical features on the duration of antibiotic therapy in critically ill infected patients, compared to a control group treated according to the best available evidence for rational antibiotic treatment in this population.

\section{Methods}

\section{Study design}

This was an open-label, randomized, parallel-group trial conducted in two intensive care units (ICUs) of the Hospital das Clínicas da Universidade Federal de Minas Gerais (UFMG) between January 2017 and May 2018, in Belo Horizonte, Brazil [20]. The study was enrolled in
ClinicalTrials.gov (NCT02987790) and approved by the ethics committee of the home institution.

\section{Participants and randomization}

All adult patients (age $\geq 18$ years) admitted to the ICUs were considered for potential inclusion according to clinical suspicion or microbiological confirmation of infection and the prospect of an ICU stay longer than 24 h. The diagnosis of sepsis or septic shock was considered according to current definitions [21]. The exclusion criteria were patients using antibiotics for more than $48 \mathrm{~h}$ at the time of screening, severe immunosuppression, patients under full and exclusive palliative care, death expectancy for the next $24 \mathrm{~h}$, diagnose of infections known to require prolonged antibiotic therapy, and patients that underwent major surgery in the previous 5 days. For details of definitions and justifications of inclusion and exclusion criteria, see Additional file 1.

Eligible patients were randomly assigned to one of two groups: a CRP-guided therapy or a control group. Randomization was performed individually at an allocation rate of $1: 1$, using a computer-generated random number table that was sequenced in enumerated and sealed brown envelopes. The random allocation sequence was generated and supervised by a researcher not involved with the inclusion, follow-up, or analysis of the data. Patients were screened, randomized, and assigned to the groups by the principal investigator and assistants. Due to the nature of the intervention, the investigators and the assistant physicians were aware of the group in which the patients had been included. Preparation and conduction of the study followed the recommendations of the CONSORT Statement [22].

\section{Intervention}

The main purpose of this study was to evaluate if a strategy of CRP-guided therapy adds value to an already rational clinical practice of antibiotic use, applied to all study participants. Accordingly, in the control group, decisions about treatment time were taken according to the best evidence established in the literature for duration of antibiotic treatment [1-6]. The study protocol provided that antibiotic therapy should be discontinued in the control group when the patient reached clinical 
criteria for suspension, which did not include CRP levels and was based on clinical improvement, microbiological results, and stipulated time according to infectious focus. This stipulated duration is objectively described in Additional file 2. Apart from the values used in the diagnostic workup for infection, CRP levels were not provided to the clinical assistants and research teams during the antibiotic therapy period in this group of patients.

In the intervention group, the duration of antibiotic therapy was defined through a clinical protocol based on the daily serum levels of CRP and patient's clinical evolution (Fig. 1). The researchers recommended discontinuation of antibiotic treatment when the criteria in the study protocol were obtained. For patients with persistently elevated CRP serum levels but with clinical improvement and absence of signs of active infection, the duration of antibiotic therapy was the same as that suggested for the control group.

For both groups, the study protocol determined that patients with bacteremia received at least five full days of adequate antibiotic coverage. In all cases included, a strategy regarding the antibiotic therapy was recommended to the ICU teams. However, any decision on antimicrobial suspension was ultimately the responsibility of clinical assistants, who were allowed to keep or interrupt antibiotics at their discretion in both groups. We considered "non-adherence to the protocol" any case in where the treating team chose not to follow the investigators' recommendations, either stopping antibiotics earlier or later than recommended by the protocol. The study protocol established longer antibiotic treatments independently of the levels of the biomarker (in the intervention group) or of the pre-determined time (in the control group) in cases of unfavorable clinical evolution, maintenance of uncontrolled focus, and clinical or microbiological findings that require extended antibiotic therapies. These cases were monitored daily by the research team, who recommended discontinuation of antibiotics as soon as possible. For both groups, a strategy of antibiotic de-escalation was recommended whenever possible.

\section{Outcomes and data collection}

Patients were followed up by researchers from the time of inclusion until hospital discharge, death, or up to 90th day, whichever occurred first. Each patient was included only once.

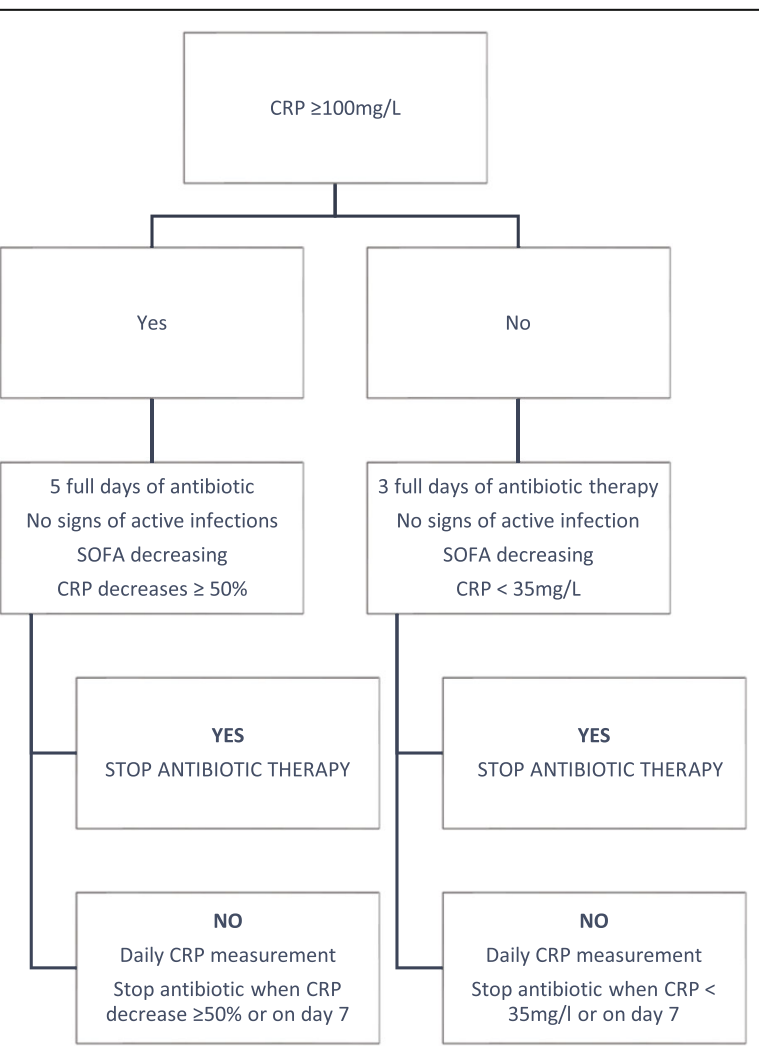

ICU - Intensive care unit; CRP - C reactive protein; SOFA - Sequential organ failure assessment.

Fig. 1 Decision-making flowchart for antibiotic discontinuation based on CRP levels. ICU, intensive care unit; CRP, C-reactive protein; SOFA, Sequential Organ Failure Assessment 
The primary outcome analyzed was the duration of antibiotic therapy of the index infection episode. The sample size calculation was done with $5 \%$ alpha error and $80 \%$ power. A previous study comparing the duration of antibiotic therapy in septic patients under the guidance of CRP versus PCT revealed that the mean duration of treatment was $7.2 \pm 3.5$ days for the CRP group and $8.1 \pm 3.7$ days for the PCT group [19]. For the present study, the expected mean for the control group was estimated from the mean observed in the PCT group in the cited study ( $\sim 8$ days), and the expected days of treatment in the CRP group was reduced to 6 days, keeping the standard deviations (SDs) found and obtaining an effect size of 0.55 by $t$ test for independent samples. Thus, we estimated the need for 53 patients per group, plus a $15 \%$ correction due to a non-normal distribution of data, totaling the sample size as 122 patients. Detailed calculation is described in Additional file 3.

Secondary outcomes were mortality for any cause on intensive care and at the 28th day, frequency of therapeutic failure and recurrence of infections, frequency of sequential nosocomial infections, the occurrence of sequential infections by multi-resistant bacteria, time in mechanical ventilation, and length of in hospital and intensive care stay. The definitions adopted for the response variables are described in Additional file 4.

The registry of the laboratory and clinical information was carried out prospectively. The data included demographic information; the scores Charlson [23], Sequential Organ Failure Assessment (SOFA) [21], Simplified Acute Physiology Score 3 (SAPS 3) [24], and Acute Physiology and Chronic Health Evaluation II (APACHE II) [25]; sepsis classification [21]; infections types and microbiology [26]; and choice of antimicrobials and respective length of use. CRP was measured in the serum at the inclusion date and daily within the first 7 days of follow-up, using the test for quantitative determination of serum CRP concentration (Vitros-Johnson \& Johnson, USA).

\section{Statistical analyses}

All analyses were performed using $\mathrm{R}$ version 3.1.1 ( $\mathrm{R}$ Foundation for statistical computing, Vienna, Austria) and SPSS (SPSS Statistics 20.0, Armonk, NY: IBM Corp. USA). Categorical variables are presented according to their absolute and relative frequencies. Continuous variables (non-normal distribution) are presented as median and interquartile range $25-75 \%(\mathrm{Q} 1-\mathrm{Q} 3)$.

Primary and secondary outcomes were primarily analyzed according to intention to treat (ITT). Both groups were compared using chi-square or Student's $t$ test/ Mann-Whitney $U$ test as indicated. For additional analysis of the primary outcomes, a cumulative curve of antibiotic discontinuation was compared between both groups (time-to-event analysis) using the Wilcoxon test [27]. When relevant, the results are presented by odds ratio and 95\% confidence intervals. Two-tailed tests and a significance level of 0.05 were used for all analyses.

\section{Results}

Baseline demographics and clinical characteristics

We evaluated 437 patients for eligibility, and a total of 135 patients were randomized. After randomization, five patients were excluded, and 130 patients were included in the intention-to-treat analysis (Fig. 2).

Demographics and clinical characteristics at inclusion were similar between patients of the two groups (Table 1). The established empiric antimicrobial therapy was adequate in $90 \%$ of the cases [28]. The values of CRP upon admission and during the first 10 days of follow-up did not reveal significant differences between the groups (Additional file 5).

\section{Outcome results: intention-to-treat analysis}

The ITT analysis revealed that the median duration of antibiotic therapy in the index episode of infection was similar in both groups, with first and third quartiles showing higher values in the control group: 7 (5-8.8) days in the CRP group versus 7 (7-11.3) days in the control group ( $p=0.011)$ (Table 2; Additional file 6). In the CRP group, more patients had their antimicrobial therapy suspended up to the fifth day of follow-up compared to the control (35.9\% vs. $10.6 \%$, OR 4.7, 95\% CI 1.9-12, $p=0.001)$. The proportion of patients taking antibiotic therapy over the first 14 days of follow-up is presented in Fig. 3.

There was no between-group significant difference regarding the total days of antibiotic exposure during follow-up and antibiotic-free days. Similar results were found for ICU and hospital length of stay, mechanical ventilation time, intensive care and 28th-day mortality, sepsis-related death, recurrence of infection, new nosocomial infections, and infection with multidrug-resistant bacteria (Table 2).

In a time-to-event data analysis, with the target event defined as "antibiotic suspension" and censoring patients who did not experience this outcome (due to death or hospital discharge in use of antibiotic therapy), a significant lower antibiotic exposure for the index infection episode was observed in the CRP group $(p=0.007)$ (Fig. 4).

The workflow of interventions in each subgroup completed with outcome related to each of the subpopulations is depicted in Additional file 7.

\section{Outcome results: per-protocol and subgroup analysis}

The rate of adherence to the protocol was 91\% (92.2\% CRP vs. $89.4 \%$ control, $p=0.764)$. Analysis per protocol 


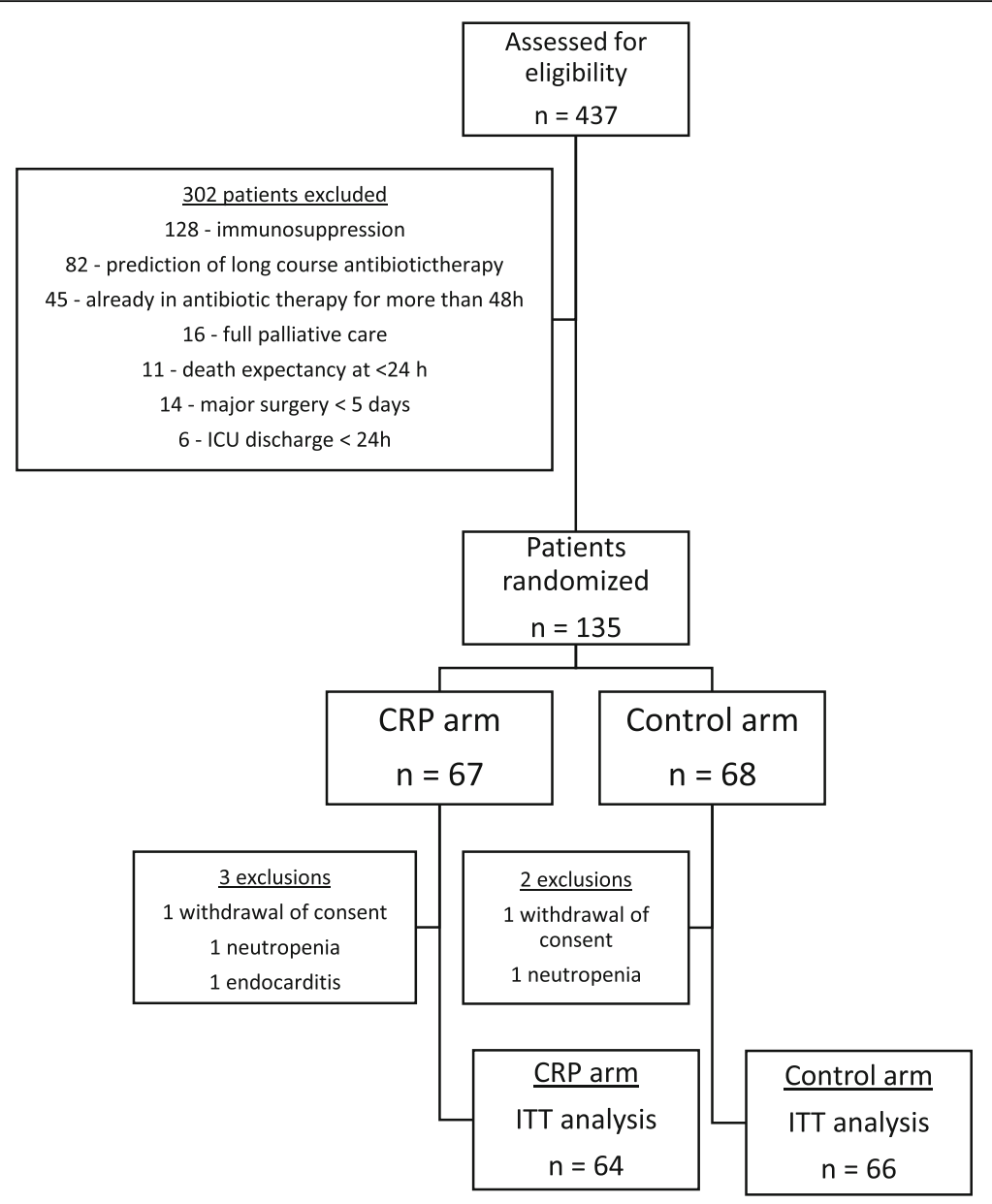

Fig. 2 Inclusion flow diagram

revealed a reduction of 1 day in the median duration of antibiotic therapy for the index infection episode in the CRP group in comparison to the control (6 (5-8) days vs. $7(7-10)$ days; $p=0.011$ ) (Additional file 8). Similar results were observed in post hoc analysis restricted to patients with SAPS 3 less than or equal to $59(n=66)$ at admission, patients with community-acquired infections $(n=56)$, patients with lower-respiratory tract infections $(n=58)$, and those who had adequate initial empirical antimicrobial therapy $(n=117)$ (Additional file 8).

\section{Discussion}

In this randomized clinical trial, we investigated the usefulness of a CRP-based protocol to reduce the duration of antibiotic therapy in critically ill patients undergoing an evidence-based judicious use of antibiotics strategy. We found lower antibiotic exposure in the intervention patients in comparison to controls, who were treated according to the best practice in antibiotic therapy $[1-3,5$, $8]$, only when considering the index infection episode. For this first treated episode, despite a similar median time of therapy, there was a narrower distribution of this parameter in the CRP arm patients. Moreover, in the CRP group, more patients had their antimicrobial therapy suspended up to the fifth day of follow-up, with a significant lower antibiotic exposure in the time-to-event analysis. Finally, the analysis per protocol revealed a reduction of 1 day in the median duration of antibiotic therapy in the intervention group. It should be stressed that these findings did not translate into more antibiotic-free days or in a reduced antimicrobial exposure.

Given the benefits offered by the rational use of antibiotics, including the reduction of multiresistant bacteria $[5,29,30]$, treatment costs [31], frequency of adverse effects [32], and less interference with microbiome, objective criteria to define the ideal treatment length is warranted. CRP is a low-cost and affordable biomarker [10], routinely used in intensive care, that has been shown relate to prognosis in studies involving different populations with serious infectious conditions [17, 20, 33, 34]. 
Table 1 Baseline demographic and clinical characteristics of the population

\begin{tabular}{|c|c|c|c|c|}
\hline Characteristics & Overall $(n=130)$ & CRP group $(n=64)$ & Control $(n=66)$ & $p$ value \\
\hline Age, years (median, Q1-Q3) & $61(51-68)$ & $62(53-68)$ & $60(49-70)$ & 0.513 \\
\hline Age, years (mean, SD) & $58.6( \pm 15.8)$ & $60.2( \pm 14)$ & $57( \pm 17.3)$ & 0.252 \\
\hline Female sex, $n(\%)$ & $62(47.7 \%)$ & $30(46.9 \%)$ & $32(48.5 \%)$ & 0.854 \\
\hline Medical patient, $n$ (\%) & $107(82.3 \%)$ & $53(82.8 \%)$ & $54(81.8 \%)$ & 0.882 \\
\hline \multicolumn{5}{|l|}{ Comorbidities, $n(\%)$} \\
\hline Heart failure & $26(20 \%)$ & $14(21.9 \%)$ & $12(18.2 \%)$ & 0.599 \\
\hline Solid malignancy & $15(11.5 \%)$ & 7 (10.9\%) & $8(12.1 \%)$ & 0.934 \\
\hline Hematologic malignancy & $2(1.5 \%)$ & $1(1.6 \%)$ & $1(1.5 \%)$ & 1.0 \\
\hline COPD & $13(10 \%)$ & $8(12.5 \%)$ & $5(7.6 \%)$ & 0.348 \\
\hline Cirrhosis & $14(10.8 \%)$ & $8(12.5 \%)$ & $6(9.1 \%)$ & 0.531 \\
\hline Chronic renal failure & $20(15.4 \%)$ & $11(17.2 \%)$ & $9(13.6 \%)$ & 0.575 \\
\hline Dialytic chronic renal failure & $8(6.2 \%)$ & $5(7.8 \%)$ & $3(4.5 \%)$ & 0.438 \\
\hline Hypertension & $69(53.1 \%)$ & $38(59.4 \%)$ & $31(47 \%)$ & 0.157 \\
\hline Diabetes & $44(33.8 \%)$ & $25(39.1 \%)$ & $19(28.8 \%)$ & 0.216 \\
\hline PLWHA & $1(0.8 \%)$ & $1(1.6 \%)$ & $0(0 \%)$ & 0.308 \\
\hline Charlson (median, Q1-Q3) & $4(2-5)$ & $4(2-5)$ & $3(1.8-6)$ & 0.126 \\
\hline SAPS 3 (median, Q1-Q3) & $59(50-70)$ & $62(49-70)$ & $59(51-70)$ & 0.119 \\
\hline APACHE II (median, Q1-Q3) & $18(13-22)$ & $18(14-23)$ & $16(13-21)$ & 0.909 \\
\hline SOFA (median, Q1-Q3) & $7(5-9)$ & $7(4-9)$ & $6(5-9)$ & 0.356 \\
\hline Respiration & $2(1-2)$ & $2(1-2)$ & $2(1-2)$ & 0.618 \\
\hline Coagulation & $0(0-1)$ & $0(0-2)$ & $0(0-1)$ & 0.175 \\
\hline Liver & $0(0-1)$ & $0(0-1)$ & $0(0-1)$ & 0.584 \\
\hline Cardiovascular & $1(0-4)$ & $1(0-4)$ & $2(0-4)$ & 0.458 \\
\hline CNS & $1(0-2)$ & $1(0-2)$ & $0(0-1)$ & 0.071 \\
\hline Renal & $1(0-3)$ & $1(0-4)$ & $1(0-3)$ & 0.678 \\
\hline Sepsis classification, $n(\%)$ & & & & 0.502 \\
\hline Infection & $8(6.2 \%)$ & $5(7.8 \%)$ & $3(4.5 \%)$ & \\
\hline Sepsis & $80(61.5 \%)$ & $41(64.1 \%)$ & $39(59.1 \%)$ & \\
\hline Septic shock & $42(32.3 \%)$ & $18(28.1 \%)$ & $24(36.4 \%)$ & \\
\hline First infection episode, $n$ (\%) & $115(88.5 \%)$ & $54(84.4 \%)$ & $61(92.4 \%)$ & 0.151 \\
\hline Site of infection, $n$ (\%) & & & & 0.228 \\
\hline Lungs & $58(44.6 \%)$ & $27(42.2 \%)$ & 31 (47\%) & \\
\hline Abdomen & $29(22.3 \%)$ & $13(20.3 \%)$ & $16(24.2 \%)$ & \\
\hline Urine & $20(15.4 \%)$ & $8(12.5 \%)$ & $12(18.2 \%)$ & \\
\hline Catheter & $6(4.6 \%)$ & $3(4.7 \%)$ & $3(4.5 \%)$ & \\
\hline Skin and soft tissue & $5(3.8 \%)$ & $5(7.8 \%)$ & $0(0 \%)$ & \\
\hline CNS & $2(1.5 \%)$ & $2(3.1 \%)$ & $0(0 \%)$ & \\
\hline Others & $10(7.7 \%)$ & $6(9.4 \%)$ & $4(6 \%)$ & \\
\hline Nosocomial infection, $n$ (\%) & $74(57 \%)$ & 39 (61\%) & 35 (53\%) & 0.363 \\
\hline Positive microbiology, $n$ (\%) & $66(50.8 \%)$ & $29(45.3 \%)$ & $37(56.1 \%)$ & 0.220 \\
\hline Positive blood culture, $n(\%)$ & $40(30.8 \%)$ & $16(25 \%)$ & $24(36.4 \%)$ & 0.160 \\
\hline MDR infections, $n(\%)$ & $29(22.3 \%)$ & $17(26.5 \%)$ & $12(18.2 \%)$ & 0.251 \\
\hline Appropriate empirical therapy, $n(\%)$ & $117(90 \%)$ & $58(90.6 \%)$ & $59(89.4 \%)$ & 0.815 \\
\hline Ventilatory support first $72 \mathrm{~h}, n$ (\%) & $77(59.2 \%)$ & $39(60.9 \%)$ & $38(57.6 \%)$ & 0.697 \\
\hline
\end{tabular}


Table 1 Baseline demographic and clinical characteristics of the population (Continued)

\begin{tabular}{|c|c|c|c|c|}
\hline Characteristics & Overall $(n=130)$ & CRP group $(n=64)$ & Control $(n=66)$ & $p$ value \\
\hline Hemodialysis first $72 \mathrm{~h}, n(\%)$ & $33(25.4 \%)$ & $18(28.1 \%)$ & $15(22.7 \%)$ & 0.480 \\
\hline Inotropes first $72 \mathrm{~h}, n$ (\%) & $16(12.3 \%)$ & $9(14.1 \%)$ & $7(10.6 \%)$ & 0.549 \\
\hline Steroids first $72 \mathrm{~h}, \boldsymbol{n}$ (\%) & $27(20.8 \%)$ & $16(25 \%)$ & $11(16.7 \%)$ & 0.242 \\
\hline Steroids for septic shock, $n$ (\%) & $4(3 \%)$ & $2(3.1 \%)$ & $2(3 \%)$ & 0.369 \\
\hline Vasopressor first $72 \mathrm{~h}, n$ (\%) & $62(47.7 \%)$ & $31(48.4 \%)$ & $31(47 \%)$ & 0.867 \\
\hline Lactate mg/dl (median, Q1-Q3) & $2(1.5-2.8)$ & $1.9(1.4-2.4)$ & $2(1.6-3)$ & 0.599 \\
\hline Leucocytes $\mathrm{g} / \mathrm{dl} \times 10^{3}$ (median, Q1-Q3) & $12(8.7-16.8)$ & $11.7(8-15.1)$ & $12.6(9-17.8)$ & 0.180 \\
\hline Neutrophil g/dl $\times 10^{3}$ (median, Q1-Q3) & $8.7(6-13.3)$ & $8.7(5.3-12.3)$ & $8.8(6-14.4)$ & 0.591 \\
\hline CRP mg/L (median, Q1-Q3) & $227(137-334$ & $199(75-313)$ & $234(151-332)$ & 0.095 \\
\hline
\end{tabular}

COPD chronic obstructive pulmonary disease, PLWAH people living with HIV and AIDS, SAPS-3 Simplified Acute Physiology Score 3, APACHE II Acute Physiology and Chronic Health Disease Classification System II, SOFA Sequential Sepsis-Related Organ Failure Assessment, CNS central nervous System, CRP C-reactive protein

Previous studies using biomarkers, notably PCT, included control groups in which the therapeutic strategy was freely determined by the assistant team. This strategy may have led to excessively long treatment duration of the control groups [10], which varied from 10 [11] to 15 days [12]. A meta-analysis involving data from more than 4000 patients on PCT-guided antibiotic therapy of acute respiratory infections revealed that the PCTguided group was treated for 7 days in comparison with the control, which received 10 days treatment, or 14 days for patients in intensive care settings [35]. In our study, we used the best standard of care in the control group, not the usual care as described above.

More recently, two studies have tested the usefulness of biomarker-guided antibiotic therapy compared to controls using shorter therapies. In a single-center study, Oliveira et al. found that a PCT-based protocol was not superior to a protocol based on serum CRP levels for reducing the use of antibiotics in sepsis. It is worth highlighting the fact that in this study, the researchers originally stipulated a maximum of 7 days for the duration of the therapy, independently of the levels of biomarkers [19]. In a larger Dutch study, de Jong et al. showed the usefulness of PCT to reduce the duration of antibiotic therapy in critically ill patients, with 5 days as the median treatment time compared to 7 days for controls [14]. It is noteworthy that the population included in the present work was significantly more severely ill than the patients included in the Dutch study [14] (septic shock 32.3\% vs. $18.5 \%$, respectively), which reinforces the value of our results, even if incipient.

Table 2 Primary and secondary endpoints by treatment arm in the intention-to-treat analysis

\begin{tabular}{|c|c|c|c|c|}
\hline Outcomes & Overall $(n=130)$ & CRP group $(n=64)$ & Control $(n=66)$ & $p$ value \\
\hline \multicolumn{5}{|l|}{ Primary outcomes } \\
\hline Duration of antibiotic therapy (median, Q1-Q3) & $7(5-10)$ & $7(5-8.8)$ & $7(7-11.3)$ & 0.011 \\
\hline Duration of antibiotic therapy (mean, $\pm \mathrm{SD}$ ) & $9( \pm 8)$ & $8( \pm 6.3)$ & $10( \pm 9.3)$ & 0.264 \\
\hline \multicolumn{5}{|l|}{ Secondary outcomes } \\
\hline Total exposure to antibiotic, days (median, Q1-Q3) & $8(7-17)$ & $8(6-19)$ & $8.5(7-16)$ & 0.564 \\
\hline Antibiotic-free period, days/100 live days (median, Q1-Q3) & $47.5(15.1-63.1)$ & $51.6(12.9-67.2)$ & $40.6(18.8-59.3)$ & 0.252 \\
\hline De-escalation rate (\%) & $40(30.7 \%)$ & $19(29.7 \%)$ & $21(31.8)$ & 0.850 \\
\hline Length of stay in ICU, days (median, Q1-Q3) & $8(4-15)$ & $8(4-15)$ & $8(4-17)$ & 0.414 \\
\hline Length of stay in hospital, days (median, Q1-Q3) & $29(15-47)$ & $31.5(16-53)$ & $25.5(15-43)$ & 0.356 \\
\hline Length of mechanical ventilation support, days (median, Q1-Q3) & $2.5(0-9)$ & $2(0-9)$ & $3(0-9)$ & 0.676 \\
\hline 28th-day mortality, n (\%) & $33(25.4 \%)$ & $18(28.1 \%)$ & $15(22.7 \%)$ & 0.480 \\
\hline ICU mortality, $n(\%)$ & $24(18.5 \%)$ & $12(18.8 \%)$ & $12(18.2 \%)$ & 0.933 \\
\hline Sepsis-related death, $n(\%)$ & $25(19.2 \%)$ & $15(23.4 \%)$ & $10(15.2 \%)$ & 0.363 \\
\hline Recurrence of first infection, $n(\%)$ & $4(3.1 \%)$ & $3(4.7 \%)$ & $1(1.5 \%)$ & 0.295 \\
\hline Sequential nosocomial infection, $n(\%)$ & $43(33.1 \%)$ & $21(32.8 \%)$ & $22(33.3 \%)$ & 0.950 \\
\hline MDR pathogen infection, $n(\%)$ & $18(13.8 \%)$ & $9(14.1 \%)$ & $9(13.6 \%)$ & 0.572 \\
\hline
\end{tabular}


Proportion of patients (\%) on antibiotics during the first 14 days of follow-up

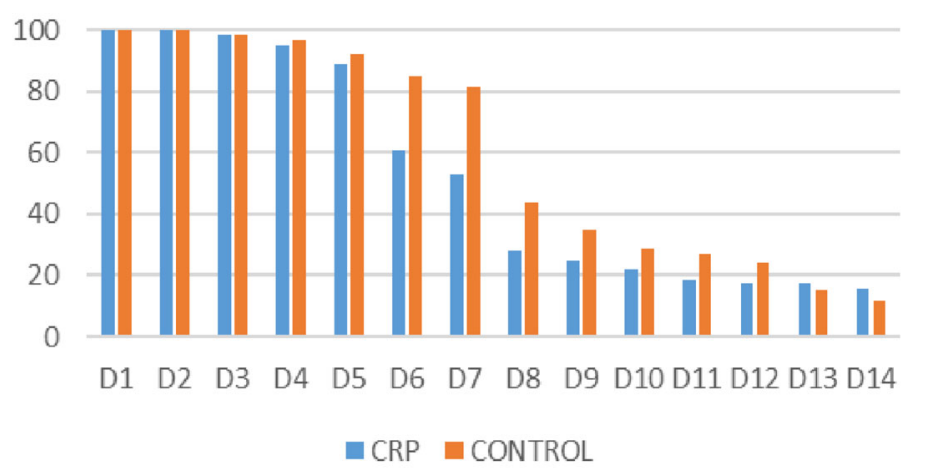

P-value for comparison of frequency between groups by the chi-square test:

\begin{tabular}{lll} 
Follow-up Day & OR $(95 \%$ IC) & p value \\
\hline Day 1 & $*$ & $*$ \\
Day 2 & $*$ & $*$ \\
Day 3 & $0.969(0.059-15.834)$ & 0.983 \\
Day 4 & $0.635(0.103-3.934)$ & 0.623 \\
Day 5 & $0.667(0.2-2.223)$ & 0.508 \\
Day 6 & $0.279(0.12-0.654)$ & $\mathbf{0 . 0 0 2}$ \\
Day 7 & $0.252(0.114-0.558)$ & $<0.001$ \\
Day 8 & $0.499(0.241-1.036)$ & 0.061 \\
Day 9 & $0.623(0.292-1.332)$ & 0.221 \\
Day 10 & $0.693(0.312-1.537)$ & 0.365 \\
Day 11 & $0.615(0.269-1.410)$ & 0.249 \\
Day 12 & $0.649(0.275-1.532)$ & 0.322 \\
Day 13 & $1.162(0.456-2.961)$ & 0.753 \\
Day 14 & $1.343(0.493-3.653)$ & 0.563
\end{tabular}

Fig. 3 Proportion of patients (\%) on antibiotics during the first 14 days of follow-up. $p$ value for comparison of frequency between groups by the chi-square test

$$
\text { Wilcoxon }-\mathrm{p}=(0.00713)
$$
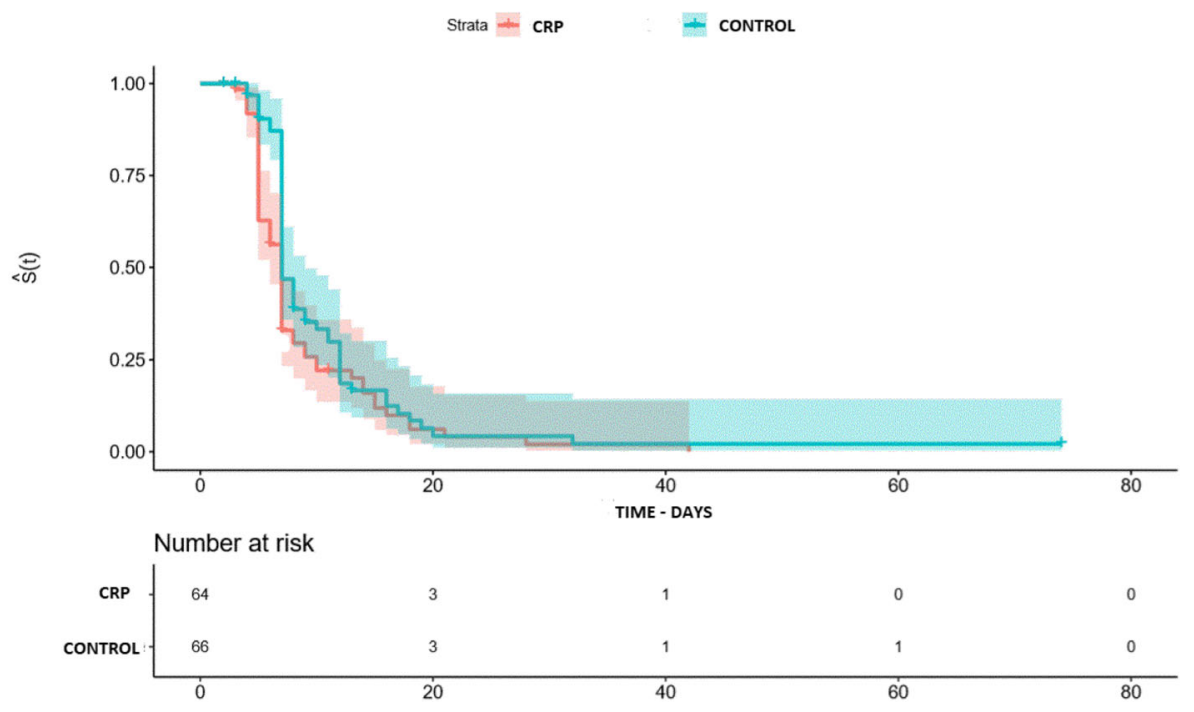

Time-to-event analysis

Fig. 4 Cumulative curve of antibiotic suspension. Time-to-event analysis 
Although our study showed no difference in median duration of antibiotics in the ITT analysis, there were more patients which received shorter durations of antibiotics in the CRP arm. Also, there was less exposure in the CRP group in the cumulative curve of antibiotic suspension for the first infection episode. Further, 1-day reduction in median duration of antibiotic treatment was found in the per-protocol analysis and in different post hoc analyses of subgroups. Specifically, in patients of lower severity and complexity (e.g., community-acquired infections and SAPS-3 $<50 \%$ ), the difference found may be justified by the easier application of the decision flowchart. In patients with respiratory tract infection, there is an already known better CRP performance in patients with pneumonia [36]. Patients who had appropriate initial empirical antibiotic therapy may have presented better results by the lower interference of inadequate initial antibiotics in treatment time [28, 37]. These preliminary findings reinforce the potential role of a CRP-guided protocol in reducing antibiotic exposure in hospitalized infected patients. Interestingly, in a recent published meta-analysis, authors found that the use of PCT algorithms to guide antibiotic therapy was associated with increased survival especially when combined with a CRP-guided strategy [16].

The rate of adherence to the protocol reported herein was higher than that reported in previous clinical trials $[13,14]$. Patients were included when in intensive care and followed up until hospital discharge or death. Therefore, interventions were also applied in other hospital units. This strategy allowed the high rates of adhesion to the protocol and proved feasible from the logistic point of view.

Despite such promising findings, other relevant investigated outcomes such as antibiotic-free days and total time of antibiotic therapy during follow-up revealed similar between groups. Also, no statistically significant differences were found in safety and survival outcomes. These findings suggest that CRP-guided therapy may be effective and safe in some specific scenarios, although further studies, with a sample size powered for safety analysis, should be conducted to confirm this hypothesis. Ideally, in settings with a less complex patient profile, where single courses of antibiotics are held more often.

Our study has several limitations that should be mentioned. This was a single-center study, restricted to two intensive care units of a high complexity hospital. Therefore, the findings lack external validity and cannot be extrapolated to other populations. In addition, the inherent open design may have biased the results, favoring the alternative hypothesis. Third, there was a high rate of non-inclusion among the patients evaluated for potential eligibility. Although this scenario has been observed in several similar studies [11, 14], this fact limits the population to which the protocol can be applied, especially immunosuppressed population. It remains unclear how these patients, including those with immunosuppressive dose corticosteroid therapy, respond to CRPguided antibiotic therapy. Also, although infection with non-fermenting Gram-negative bacteria was not an exclusion criterion, patients with this kind of infection were poorly represented in this study. Fourth, there was an apparent trend towards higher mortality in the CRP-guided therapy group, with no statistically significant difference. However, sepsis-associated mortality was quite similar between the two groups, as well as recurrent infection rates. Also, mortality rate of patients who had early suspension of antibiotics, according to study protocols, was lower than the overall mortality rate and similar between intervention and control groups (Additional file 7).

\section{Conclusions}

A protocol based on daily monitoring of CRP levels may support a tailored time of antibiotic therapy in critically ill patients in a single infection episode, but without reducing the total exposure of these patients to antimicrobials. The subtle time reduction observed in the group of patients undergoing CRP-guided therapy may be potentially impacting in less complex scenarios, since it was observed even in a scenario of judicious use of antibiotics. CRP-guided strategy is feasible. Further studies are needed to add up to these findings, to properly assess safety outcomes and to evaluate the real impact of this strategy in clinical practice.

\section{Supplementary information}

Supplementary information accompanies this paper at https://doi.org/10. 1186/s13054-020-02946-y.

Additional file 1. Inclusion and exclusion criteria.

Additional file 2. Antibiotic therapy time provided for the control (best practice) group.

Additional file 3. Sample size calculation.

Additional file 4. Definitions of the response variables.

Additional file $\mathbf{5}$. Serial measurements of C-reactive protein concentrations in both study groups.

Additional file 6. Days of antibiotic therapy in the index infection episode according to the inclusion group - Intention-to-treat analysis.

Additional file 7. Workflow of interventions and outcomes.

Additional file 8. Duration of antibiotic therapy for the index infectious episode: analysis per protocol and other subgroups.

\section{Abbreviations}

APACHE II: Acute Physiology and Chronic Health Evaluation II; CAP: Community-acquired pneumonia; Cl: Confidence interval; CRP: Creactive protein; ICU: Intensive care unit; ITT: Intention to treat; OR: Odds ratio; PCT: Procalcitonin; Q1-Q3: Interquartile range; SAPS 3: Simplified Acute Physiology Score 3; SOFA: Sequential Organ Failure Assessment; UFMG: Universidade Federal de Minas Gerais; VAP: Ventilator-associated pneumonia 


\section{Acknowledgements}

We thank the ICUs staff for the routine support. We also thank the students, Andrea Jerusa, Arthur Farnese, Lucas Zica, Monize Santana, Rafael Bergo, and Rafael Carneiro, for the assistance in the inclusion process and collection of clinical data and biological material. We thank Larissa Martins and Enrico Colosimo for assistance with statistical analysis and Cecilia Ravetti and Vandack Nobre for the conceptual contributions, for the daily assistance, and for critically reviewing the paper. Finally, we would like to show our gratitude to Dr. Pedro Póvoa and Dr. Jorge Salluh for their comments that greatly improved this manuscript.

\section{Authors' contributions}

The authors read and approved the final manuscript.

\section{Funding}

This work was partially funded by the Fundação de Apoio à Pesquisa do Estado de Minas Gerais (FAPEMIG) and the Conselho Nacional de Desenvolvimento Científico e Tecnológico (CNPq).

\section{Availability of data and materials}

The datasets used and analyzed during the current study are available from the corresponding author on reasonable request.

\section{Ethics approval and consent to participate}

The study was approved by the Ethics Committee of the Universidade Federal de Minas Gerais, approval protocol number 53351416.9.0000.5149, and all inclusions required signing of an informed consent form by the patient or surrogate.

\section{Consent for publication}

All authors consent to the publication of the manuscript in Critical Care, should the article be accepted by the Editor-in-chief upon completion of the refereeing process.

\section{Competing interests}

The authors have disclosed that they do not have any conflicts of interest.

\section{Author details}

Graduate Program in Health Sciences: Infectious Diseases and Tropical Medicine, Department of Internal Medicine, School of Medicine and Hospital das Clínicas, Universidade Federal de Minas Gerais, Belo Horizonte, Brazil. ${ }^{2}$ Departamento de Clínica Médica, $2^{\circ}$ andar Faculdade de Medicina. Av. Alfredo Balena, 190, Santa Efigênia, Belo Horizonte, Minas Gerais, Brazil. ${ }^{3}$ Graduate Program in Statistics, Department of Statistics, Universidade Federal de Minas Gerais, Belo Horizonte, Brazil. ${ }^{4}$ Graduate Program in Health Sciences: Adult Health, Department of Internal Medicine, School of Medicine and Hospital das Clínicas, Universidade Federal de Minas Gerais, Belo Horizonte, Brazil.

\section{Received: 17 February 2020 Accepted: 5 May 2020} Published online: 01 June 2020

\section{References}

1. Chastre J, Wolff M, Fagon JY, et al. Comparison of 8 vs 15 days of antibiotic therapy for ventilator-associated pneumonia in adults: a randomized trial. Jama. 2003;290(19):2588-98.

2. Havey TC, Fowler RA, Daneman N. Duration of antibiotic therapy for bacteremia: a systematic review and meta-analysis. Crit Care. 2009;15(6): R267.

3. Eliakim-Raz N, Yahav D, Paul M, Leibovici L. Duration of antibiotic treatment for acute pyelonephritis and septic urinary tract infection-- 7 days or less versus longer treatment: systematic review and meta-analysis of randomized controlled trials. J Antimicrob Chemother. 2013;68(10):2183-91.

4. Onakpoya IJ, Walker AS, Tan PS, et al. Overview of systematic reviews assessing the evidence for shorter versus longer duration antibiotic treatment for bacterial infections in secondary care. PLoS One. 2018;13(3): e0194858.

5. Rattan R, Allen CJ, Sawyer RG, et al. Patients with complicated intraabdominal infection presenting with sepsis do not require longer duration of antimicrobial therapy. J Am Coll Surg. 2016;222(4):440-6.
6. Tansarli GS, Mylonakis E. Systematic review and meta-analysis of the efficacy of short-course antibiotic treatments for community-acquired pneumonia in adults. Antimicrob Agents Chemother. 2018;62(9):e00635-18.

7. Vincent $\mathrm{JL}$, Bassetti $\mathrm{M}$, François $\mathrm{B}$, et al. Advances in antibiotic therapy in the critically ill. Crit Care. 2016;20(1):133.

8. Uranga A, Espana PP, Bilbao A, et al. Duration of antibiotic treatment in community-acquired pneumonia: a multicenter randomized clinical trial. JAMA Intern Med. 2016;176(9):1257-65.

9. Klompas M, Li L, Menchaca JT, Gruber S, Epicenters CfDCaP, Program. Ultrashort-course antibiotics for patients with suspected ventilator-associated pneumonia but minimal and stable ventilator settings. Clin Infect Dis 2017; 64(7):870-876.

10. Nora D, Salluh J, Martin-Loeches I, Povoa P. Biomarker-guided antibiotic therapy-strengths and limitations. Ann Transl Med. 2017;5(10):208.

11. Nobre V, Harbarth S, Graf JD, Rohner P, Pugin J. Use of procalcitonin to shorten antibiotic treatment duration in septic patients: a randomized trial. Am J Respir Crit Care Med. 2008;177(5):498-505.

12. Stolz D, Smyrnios N, Eggimann P, et al. Procalcitonin for reduced antibiotic exposure in ventilator-associated pneumonia: a randomised study. Eur Respir J. 2009;34(6):1364-75.

13. Bouadma L, Luyt CE, Tubach F, et al. Use of procalcitonin to reduce patients' exposure to antibiotics in intensive care units (PRORATA trial): a multicentre randomised controlled trial. Lancet. 2010;375(9713):463-74.

14. de Jong E, van Oers JA, Beishuizen A, et al. Efficacy and safety of procalcitonin guidance in reducing the duration of antibiotic treatment in critically ill patients: a randomised, controlled, open-label trial. Lancet Infect Dis. 2016;16(7):819-27.

15. Wirz $Y$, Meier MA, Bouadma L, et al. Effect of procalcitonin-guided antibiotic treatment on clinical outcomes in intensive care unit patients with infection and sepsis patients: a patient-level meta-analysis of randomized trials. Crit Care. 2018;22(1):191.

16. Pepper D, Sun J, Rhee C, et al. Procalcitonin-guided antibiotic discontinuation and mortality in critically ill adults. A systematic review and meta-analysis. Chest. 2019;155(6):1109-18.

17. Dianti M, Luna CM. Do we need biomarkers for the follow-up and shortening of antibiotic treatment duration? Curr Opin Crit Care. 2018;24(5): 361-9.

18. Pierrakos C, Vincent JL. Sepsis biomarkers: a review. Crit Care. 2010;14(1):R15.

19. Oliveira CF, Botoni FA, Oliveira CR, et al. Procalcitonin versus C-reactive protein for guiding antibiotic therapy in sepsis: a randomized trial. Crit Care Med. 2013;41(10):2336-43.

20. Calsavara AJC, Costa PA, Nobre V, Teixeira AL. Factors associated with short and long term cognitive changes in patients with sepsis. Sci Rep. 2018;8(1): 4509.

21. Singer M, Deutschman CS, Seymour CW, et al. The Third International Consensus Definitions for Sepsis and Septic Shock (Sepsis-3). JAMA. 2016; 315(8):801-10

22. Moher D, Hopewell S, Schulz KF, et al. CONSORT 2010 explanation and elaboration: updated guidelines for reporting parallel group randomised trials. BMJ. 2010;340:C869.

23. Charlson ME, Pompei P, Ales KL, MacKenzie CR. A new method of classifying prognostic comorbidity in longitudinal studies: development and validation. J Chronic Dis. 1987:40(5):373-83.

24. Moreno RP, Metnitz PG, Almeida E, et al. SAPS 3--from evaluation of the patient to evaluation of the intensive care unit. Part 2: development of a prognostic model for hospital mortality at ICU admission. Intensive Care Med. 2005;31(10):1345-55.

25. Knaus WA, Draper EA, Wagner DP, Zimmerman JE. APACHE II: a severity of disease classification system. Crit Care Med. 1985;13(10):818-29.

26. Garner JS, Jarvis WR, Emori TG, Horan TC, Hughes JM. CDC definitions for nosocomial infections, 1988. Am J Infect Control. 1988;16(3):128-40.

27. Klein JP, Moeschberger ML. Survival analysis: techniques for censored and truncated data. 2nd ed. New York ; London: Springer; 2003.

28. Kumar $A, E$ Ellis $P, A r a b i Y$, et al. Initiation of inappropriate antimicrobial therapy results in a fivefold reduction of survival in human septic shock. Chest. 2009;136(5):1237-48.

29. Thomas Z, Bandali F, Sankaranarayanan J, Reardon T, Olsen KM, Network CCPT. A multicenter evaluation of prolonged empiric antibiotic therapy in adult ICUs in the United States. Crit Care Med. 2015;43(12):2527-34.

30. Singh N, Rogers P, Atwood CW, Wagener MM, Yu VL. Short-course empiric antibiotic therapy for patients with pulmonary infiltrates in the intensive 
care unit. A proposed solution for indiscriminate antibiotic prescription. Am J Respir Crit Care Med. 2000;162(2 Pt 1):505-11.

31. D'Agata EM, Magal P, Olivier D, Ruan S, Webb GF. Modeling antibiotic resistance in hospitals: the impact of minimizing treatment duration. J Theor Biol. 2007;249(3):487-99.

32. Yusuf $E$, Van Herendael $B$, Verbrugghe $W$, et al. Emergence of antimicrobial resistance to Pseudomonas aeruginosa in the intensive care unit: association with the duration of antibiotic exposure and mode of administration. Ann Intensive Care. 2017;7(1):72.

33. Seligman R, Meisner M, Lisboa TC, et al. Decreases in procalcitonin and Creactive protein are strong predictors of survival in ventilator-associated pneumonia. Crit Care. 2006;10(5):R125.

34. Lisboa T, Seligman R, Diaz E, Rodriguez A, Teixeira PJ, Rello J. C-reactive protein correlates with bacterial load and appropriate antibiotic therapy in suspected ventilator-associated pneumonia. Crit Care Med. 2008;36(1):166-71.

35. Schuetz $\mathrm{P}$, Briel M, Christ-Crain M, et al. Procalcitonin to guide initiation and duration of antibiotic treatment in acute respiratory infections: an individual patient data meta-analysis. Clin Infect Dis. 2012;55(5):651-62.

36. Chalmers JD, Singanayagam A, Hill AT. C-reactive protein is an independent predictor of severity in community-acquired pneumonia. Am J Med. 2008; 121(3):219-25.

37. Kumar A. Systematic bias in meta-analyses of time to antimicrobial in sepsis studies. Crit Care Med. 2016;44(4):e234-5.

\section{Publisher's Note}

Springer Nature remains neutral with regard to jurisdictional claims in published maps and institutional affiliations.

Ready to submit your research? Choose BMC and benefit from:

- fast, convenient online submission

- thorough peer review by experienced researchers in your field

- rapid publication on acceptance

- support for research data, including large and complex data types

- gold Open Access which fosters wider collaboration and increased citations

- maximum visibility for your research: over $100 \mathrm{M}$ website views per year

At $\mathrm{BMC}$, research is always in progress.

Learn more biomedcentral.com/submissions 